\title{
A light at the end of the tube
}

This assortment of products includes laser beam attenuators, motorized steppers and translators, laser confocal microscopy systems, beam analysers, as well as infrared and diode laser bars and systems.

\section{WA-1500/WA-1000 Wavemeter \\ From Burleigh \\ Laser characterization instruments for accurate optical wavelength measurement}

Designed for measuring the absolute wavelength of virtually any laser at the required accuracy, Burleigh offers systems for CW lasers, pulsed lasers and OPOs, operating from the ultraviolet through the visible and into the infrared. Absolute wavelength is measured continuously and in real-time for reporting or automatic laser control. The WA-1500 Wavemeter is stated to be an accurate, continuous, real-time laser wavelength measurement system. The WA- 1000 system has most of the features of its WA-1500 counterpart, but is more affordable. These systems employ scanning Michelson, interferometer-based Wavemeter technology to determine the absolute wavelength of a test laser by comparing its interference fringe pattern with that of a builtin $\mathrm{HeNe}$ laser wavelength standard. The WA-1500 achieves a stated wavelength accuracy of \pm 0.1 p.p.m. $( \pm 0.1 \mathrm{pm}$ at $1,000 \mathrm{~nm})$; whereas the WA-1000 provides an absolute wavelength accuracy of \pm 1 p.p.m. ( $\pm 1 \mathrm{pm}$ at $1,000 \mathrm{~nm}$ ). Four versions of each model are available for ultraviolet, visible, near infrared, and infrared wavelengths. Each includes a photodetector and a beamsplitter optimized for its operational wavelength range.

Reader Enquiry No. 100

\section{LBA-300PC}

\section{From Laser Lines}

Version 2.1 of this Spiricon-produced, laser beam analysis system is now available

Now running under Windows95, 98 and NT, this latest iteration includes the addition of full GPIB remote control for use in automated applications, as well improvements to the user interface, such as mouse shortcut features and improved image handling. All of the existing functions are

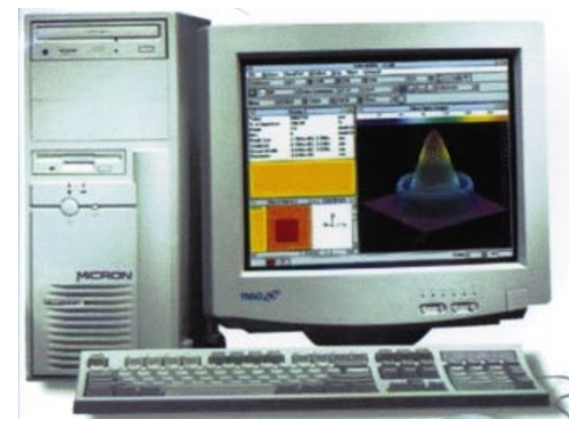

Keep your distance: remote control from Spiricon. retained, including the Ultracal automatic calibration algorithm, which is said to produce improved accuracy in laser beam diameter computations, as well as in the ISO D4 $\sigma$ (second moment) method for measuring beam widths.

Reader Enquiry No. 101

\section{Eximer laser attenuators}

From AG Electro-Optics

Acton Research's beam attenuators for the 193- $\mathrm{nm} \mathrm{ArF}$ and 248-nm KrF eximer lasers

Eximer attenuators are multi-layer dielectric-coated, fused-silica optics that provide variation in the amount of transmitted laser energy by controlling the angle of incidence on the attenuator. For example, the 193-nm attenuator can change the output power of the ArF laser from approximately 20 per cent transmission at $0^{\circ}$ (normal angle of incidence) to $85 \%$ at $45^{\circ}$ angle of incidence. The 248 -nm attenuator can vary the output power of a KrF laser from approximately ten per cent of its energy output to 90 per cent output by changing the angle of incidence of the optic from normal to $45^{\circ}$. Applications for these attenuators include excimer laser beam attenuation, beam splitting and beam monitoring.

Reader Enquiry No. 102

\section{Stepper Mike}

From L.O.T. Oriel

Stepper motors and translators for high-

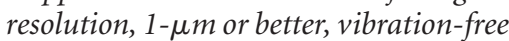
movement in small discrete steps

Offered in 1.27- and 2.54-cm travel lengths, these units operate in full-step mode at $2 \mu \mathrm{m}$ per step, or in half-step mode at 1.3 and $0.7 \mu \mathrm{m}$ per step. This stepper should prove useful for accurate, remote control in standard micrometer applications. For submicrometre translation of fibre optics, laser focusing assemblies, optical mounts or other optical components, the manufacturer offers a motorized horizontal and vertical translator. Programmable singleand dual-channel controllers with RS232 and IEEE interfaces are also available.

Reader Enquiry No. 103

\section{Modular translation stage}

From New Focus

Stages with an interlocking track design

With a twist of one screw, these stages can be easily configured for multiple applications. Modular features, a low profile, a small foot-

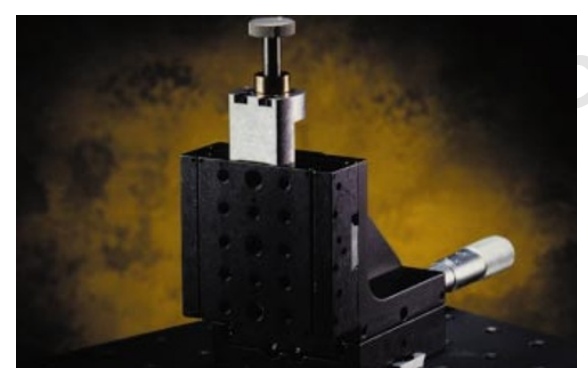

Stage management: versatility from New Focus.

print and compatibility with standard actuators make this stage compatible with most standard actuators. Being a linear stage, it should also prove useful in general-purpose optical experiments.

Reader Enquiry No. 104

\section{Laser systems}

Diode laser bars

From Coherent

Offering 40-W bars and 30-W fibre array packaged bars at $808 \mathrm{~nm}$

These bars are based on aluminium-free, active-area material and are in stock and available in a wavelength range from 800 to $820 \mathrm{~nm}$. The bars are $1-\mathrm{cm}$ linear arrays mounted on conduction-cooled packages. They do not require a special operating environment and are said to be easily integrated into existing applications. Achieving this difficult wavelength, says the manufacturer, demonstrates the company's ability to apply this technology widely. The fibrearray packaged bars offer up to $30 \mathrm{~W}$ into an $800-\mu \mathrm{m}$ fibre with a numerical aperture of $<0.16$.

Reader Enquiry No. 105

\section{PL2 $\mathrm{CO}_{2}$ lasers}

From Edinburgh Instruments

The company has extended the spectral range of these lasers through the use of isotopic gas fills

These gas mixtures can be used to increase the number of wavelengths obtainable with these PL2 $\mathrm{CO}_{2}$ lasers. The PL2 laser tube is designed to allow gas to be simply and swiftly changed, making the use of isotopic gas mixes as straightforward as standard gas mixes. Using ${ }^{13} \mathrm{C}^{16} \mathrm{O}_{2}$ is said to give output powers of $>3 \mathrm{~W}$ on the strongest lines (typically, ${ }^{13} \mathrm{C}^{16} \mathrm{O}_{2}$ has 12 lines in any of the four bands). The tunable nature of this series should prove useful in long-term studies of atmospheric conditions; and, isotopic mixtures can be chosen for coincidences with pollutant 
absorption spectra, which should prove useful in the detection and monitoring of pollution. Other uses include studies of atmospheric propagation, clear-air turbulence, high-resolution spectroscopy, optical pumping and applications that require tracking and ranging.

Reader Enquiry No. 106

\section{IMS633}

From Melles Griot

A self-contained, temperature-stabilized diode laser system

With a long coherence length and stable output, this system has been designed for holographic interferometry, and includes automatic current and temperaturecontrol electronics, as well as high-quality, beam-delivery optics. It is said to deliver 18$\mathrm{MHz}$, direct-modulation capability and high-power output in a small footprint, requiring only $5-\mathrm{VCD}$ to operate. A frontpanel C-mount is included for beam expanders and spatial filters. On the outside, a rugged, sealed enclosure keeps the laser and beam-delivery optics free from environmental contaminants. Inside the laser, there is over-voltage, reverse-voltage, transient, ESD and EMI protection. Other wavelengths, output powers and beam sizes are available upon request.

Reader Enquiry No. 107

\section{ML3-T infrared laser}

From Millennium Lasers

An infrared laser that shines in the band range 9-11 $\mu \mathrm{m}$

This is a modified, low-power, industrial/ medical laser for use in scientific applications. The ML3-T produces a TEM00 output at wavelengths selectable 9 and $11 \mu \mathrm{m}$ with a typical output power per line of a few hundred milliwatts in CW mode. The laser head is some $45-\mathrm{cm}$ long with a $10-\mathrm{cm}$ cross section. It features a rugged, stabilized cavity; sealed-tube technology; frequency stability; a compact size with low weight; and a low-voltage, safety interlocking system. This laser should prove useful in environmental and remote sensing, interferometry, infrared spectrometry and injection seeding.

Reader Enquiry No. 108

\section{Solution 582 and 633}

From Detection Limit

Two new, low-cost Raman systems

These are dispersive CCD Raman systems: the model 633 uses an external 633-nm, $40-\mathrm{W}$ HeNe laser as the excitation source, whereas the 582 system employs an internal 582-nm, 150-mW, frequency-stabilized diode laser. These fully integrated systems feature fibre-optic coupling between the laser, the remote, the miniature epi-probe

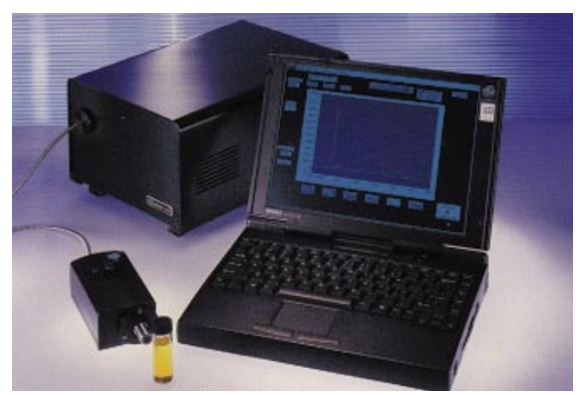

Solution 582: solves spectral analysis problems.

and the high-throughput $f / 2$ imaging spectrograph. Both systems come with a Pentium-based computer controller that is loaded with mouse-driven, LabView-based DLSPEC control and data acquisition software, which includes single-button access to the embedded Grams/32 spectral analysis and presentation software.

Reader Enquiry No. 109

\section{Gonfocalmicroscopy}

\section{MicroRadiance}

From Bio-Rad

This latest instrument is said to make confocal microscopy more accessible

Featuring a miniaturized scan head, this instrument is intended to lower the price range of this technique to make it more widely accessible. MicroRadiance has a space-saving footprint, and can also be easily transferred from one microscope to another. Compatible with many inverted, standard and fixed-stage upright microscopes, the scan head needs no user realignment as all optical components are pre-set during manufacture. Motorized elements, such as apertures and filters, are all controlled from BioRad's Lasersharp software. The MicroRadiance range consists of three upgradable models designed to accommodate individual budgets. The standard MR/A-1 can perform most single- and double-labelling fluorescence imaging techniques, as well as reflection and transmission imaging. The second model, MR1AG-1, has improved green/red doublelabelling performance via the addition of a green $\mathrm{He}-\mathrm{Ne}$ laser, which generate images with highly separated green and red channels. A further upgrade to the MR/AG-2 allows simultaneous green/red imaging through two PMT detectors.

Reader Enquiry No. 110

\section{LSM 510}

From Carl Zeiss

A compact confocal module for laser scanning microscopes with maximum spatial resolution

This fifth-generation laser scanning microscope can be combined with the high performance, fully motorized Axioplan 2 and Axiovert microscopes to form both upright and inverted laser microscope systems. The compact design is achieved using a high level of system integration and the shortest possible light paths to ensure high optical precision and stability with flexibility. Six detectors are integrated into an extremely small space, each has four confocal channels with individual computer-controlled, opticalspatial filter. Maximum spatial resolution is achieved through 2,048 $\times 2,048$ pixels and simultaneous 12-bit AD conversion for up to 4,096 brightness levels. The LSM 510 can be integrated or retrofitted to existing motorized microscopes. It runs multi-user software through Windows NT, allowing personal application programs or routine macros to be built into the system. Functions include integration, oversampling and quasi-photon counting facilities.

Reader Enquiry No. 111

These notes are compiled by Brendan Horton

from information provided by the manufacturers. For more details, fill in the reader service card bound inside the journal.

ANNOUNCEMENT

\section{Software reviews at wwwhature.com}

Turn to Nature's website where the first in a series of comparative reviews of scientific software is up and running.

\section{The Graphical Interface}

by Sharon Kardia

Nature has recruited a group of reviewers to test a variety of scientific software. The first in the series of special features has Sharon Kardia reviewing 16 graph-making packages. Each review will include a table of system functions and performance plus hyperlinks to information for contacting the software providers.

Future 'specials' will cover statistics and mathematics programs, bibliography packages and other tools of the trade.

ADVERTISEMENT

\section{Trichostatin A}

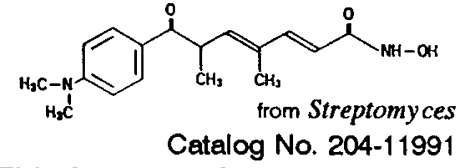

This histone deacetylase inhibitor is highly effective at low concentrations, with high specificity. For more information, please call Wako BioProducts: (800) 992-9256; (804) 271-7677. 